\title{
Effect of Zinc Incorporation on the Performance of Red Light Emitting InP Core Nanocrystals
}

Lifei Xi ${ }^{1}$, Deok-Yong Cho ${ }^{2}$, Astrid Besmehn ${ }^{3}$, Martial Duchamp ${ }^{4}$, Detlev Grützmacher ${ }^{1}$, Yeng Ming Lam ${ }^{*}$, , Beata E. Kardynat ${ }^{*}, 1$

${ }^{1}$ Semiconductor Nanoelectronics (PGI-9), Forschungszentrum Jülich, JARA-FIT, 52425 Jülich, Germany

2 IPIT \& Department of Physics, Chonbuk National University, Jeonju 561-756, Republic of Korea

3 Central Institute for Engineering, Electronics and Analytics (ZEA-3), Forschungszentrum Jülich, 52425 Jülich, Germany

${ }^{4}$ Ernst Ruska Centre, PGI-5, Forschungszentrum Jülich, JARA-FIT, 52425 Jülich, Germany

5 School of Materials Science and Engineering, Nanyang Technological University, 639798, Singapore

*Corresponding authors: YMLam@ntu.edu.sg, B.Kardnal@fz-juelich.de 


\section{Supporting Figures}

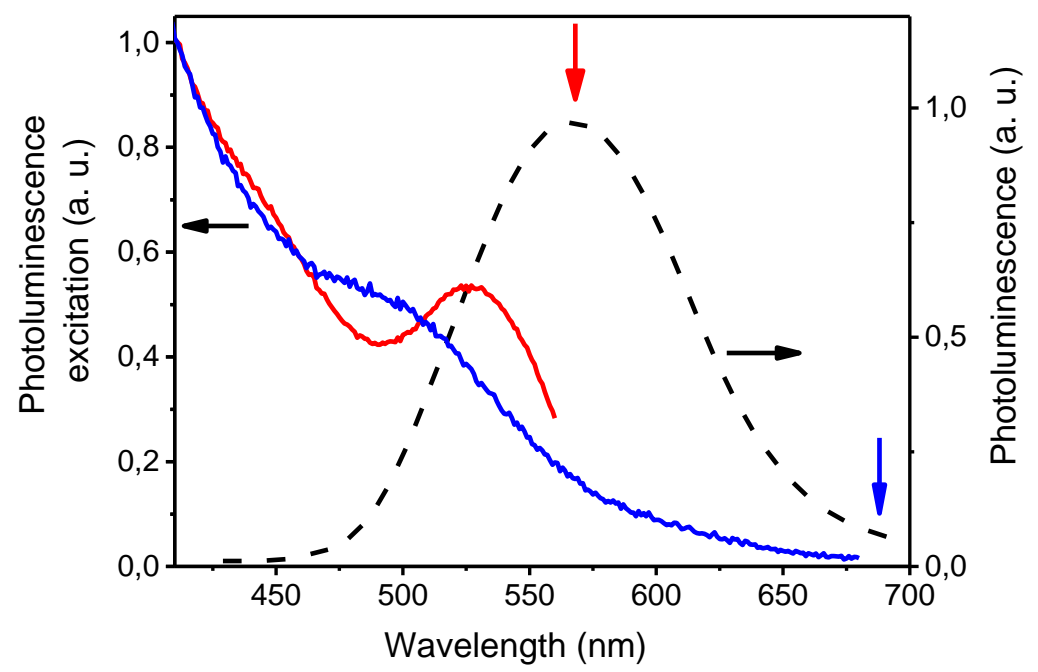

Figure S1. PL (black broken line) and PL excitation (PLE) spectra of $\operatorname{In}(\mathrm{Zn}) \mathrm{P}$ NCs cores. Two PLE spectra are included: one in which the PL emission wavelength was fixed at 568nm (red arrow, close to the central wavelength of emission) and one when emission was fixed at $690 \mathrm{~nm}$ (blue arrow, at the beginning of the PL tail). The PLE spectrum corresponding to 568nm emission (red curve) has a peak at 530nm - shorter by a Stokes shift. The PLE spectrum corresponding to $688 \mathrm{~nm}$ emission (blue curve) has a broad peak at similar wavelength range to the PLE of $568 \mathrm{~nm}$ emission. This means that photons emitted at $688 \mathrm{~nm}$ are excited at $530 \mathrm{~nm}$ in the bandto-band transition, then decay to defects states and recombine through these states. Note that due to the PL detection limit of $700 \mathrm{~nm}$, this experiment could not be conducted on the samples with peak emission of $630-650 \mathrm{~nm}$ as investigated in the main text. The behaviour presented in this figure is however generic to all samples that were investigated.
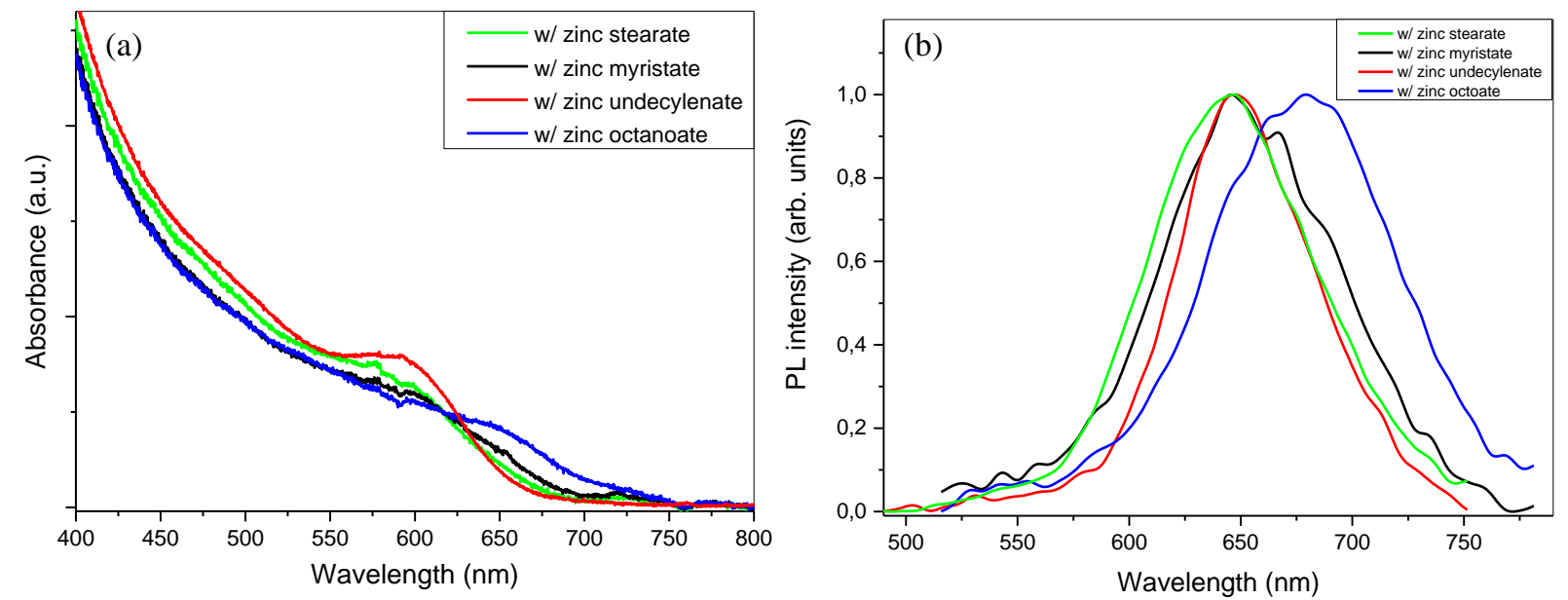

Figure S2. (a) UV-Vis and (b) normalized PL spectra of In(Zn)P NCs synthesized with different $\mathrm{Zn}$ precursors. The full width at half maximums (fwhms) of $\operatorname{In}(\mathrm{Zn}) \mathrm{P}$ NCs synthesized with zinc 
(Zn) octoate, undecylenate, myristate and stearate are $99 \pm 3,71 \pm 2,97 \pm 3$ and $90 \pm 1 \mathrm{~nm}$. It can be seen that In(Zn)P NCs synthesized with Zn undecylenate has the narrowest fwhm. Oscillations visible on the PL spectra originate from the dichroic mirror used in the detection path.
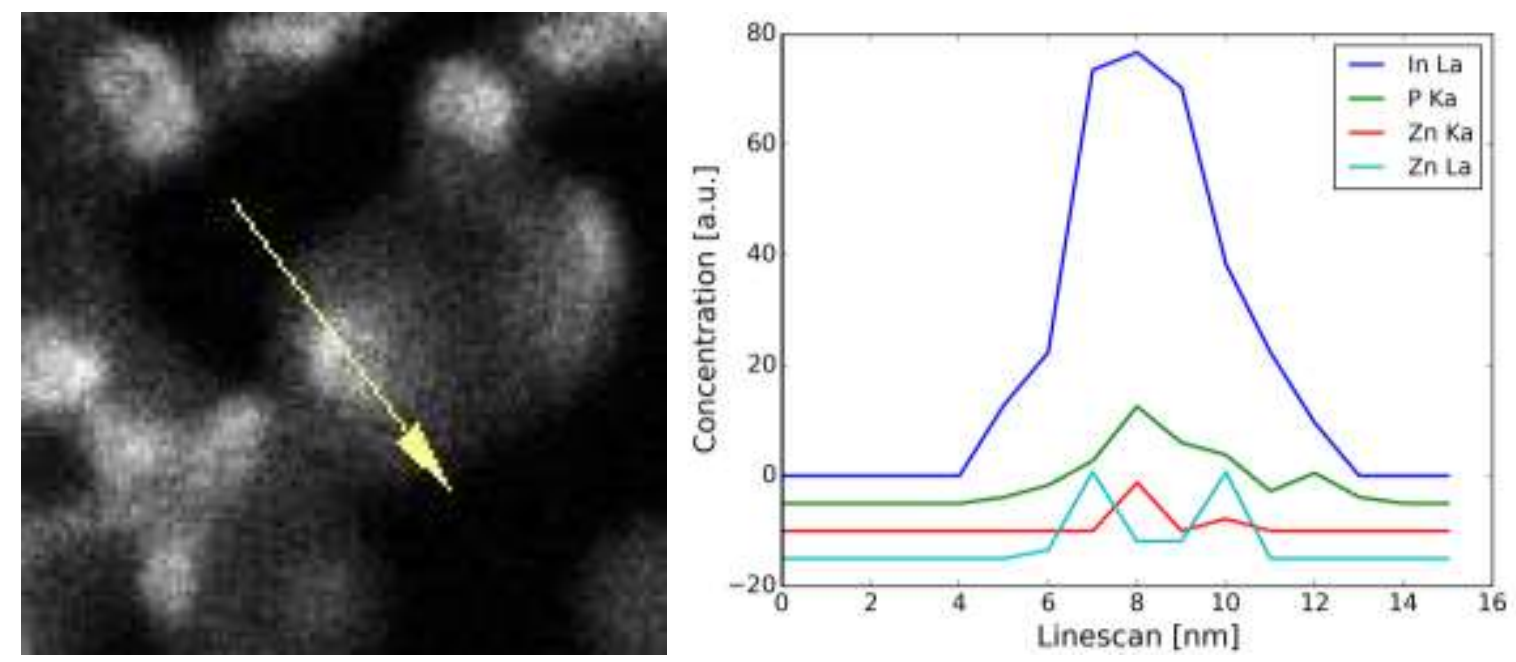

Figure S3. EDX line scan spectra of $\mathrm{In}_{\alpha^{-}}, \mathrm{Zn} \mathrm{K}_{\alpha^{-}}$and L-, and P-edges (right panel) from an individual $\mathrm{In}(\mathrm{Zn}) \mathrm{P}$ core nanocrystal (shown in the left). Spectra include Cliff Lorimer's factors and can thus be compared directly. Zn content in the NCs is estimated to be $6 \%$ from Zn $\mathrm{K}_{\alpha}$-edge and $17 \%$ from $\mathrm{Zn}$ L-edge. It was estimated as a ratio of the integrated $\mathrm{Zn} \mathrm{K} \mathrm{K}_{\alpha}-(\mathrm{Zn} \mathrm{L})$ counts to the integrated In counts. Averaging these two numbers gives $\mathrm{Zn}$ :In ratio of $11.5 \pm 5.5 \%$.

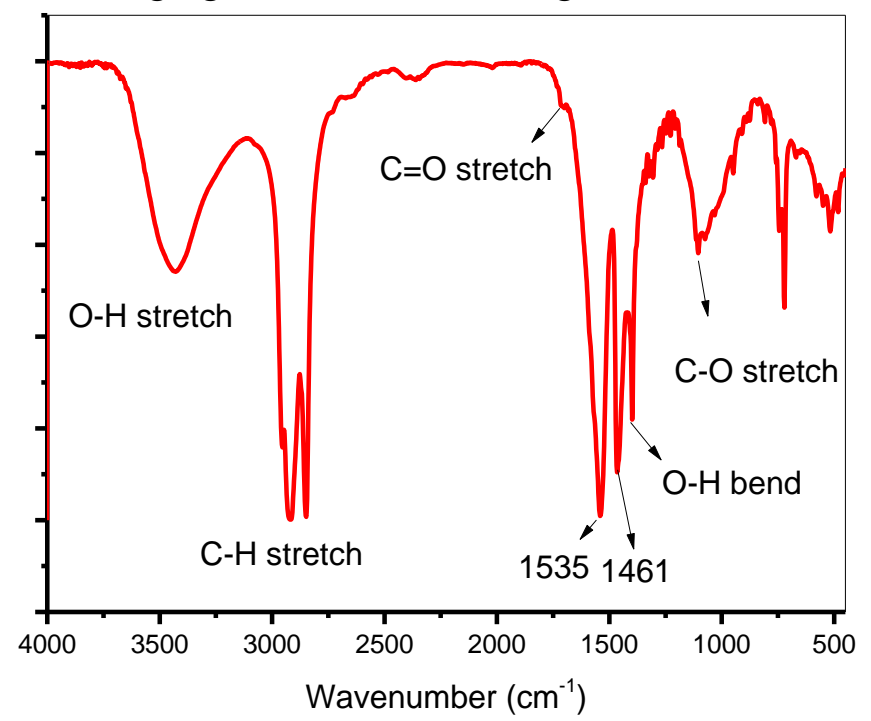

Figure S4. FT-IR spectra of In(Zn)P cores synthesized with Zn undecylenate addition. In order to characterize and probe the surface environment of the core NCs, FT-IR spectroscopy is employed. The strong vibrational bands at around 1535 and $1461 \mathrm{~cm}^{-1}$ which are respectively assigned to the antisymmetric and symmetric stretching bonds of the carboxylate ligands, undecylenate. ${ }^{5}$ This results confirms the role of carboxylate as ligand. 

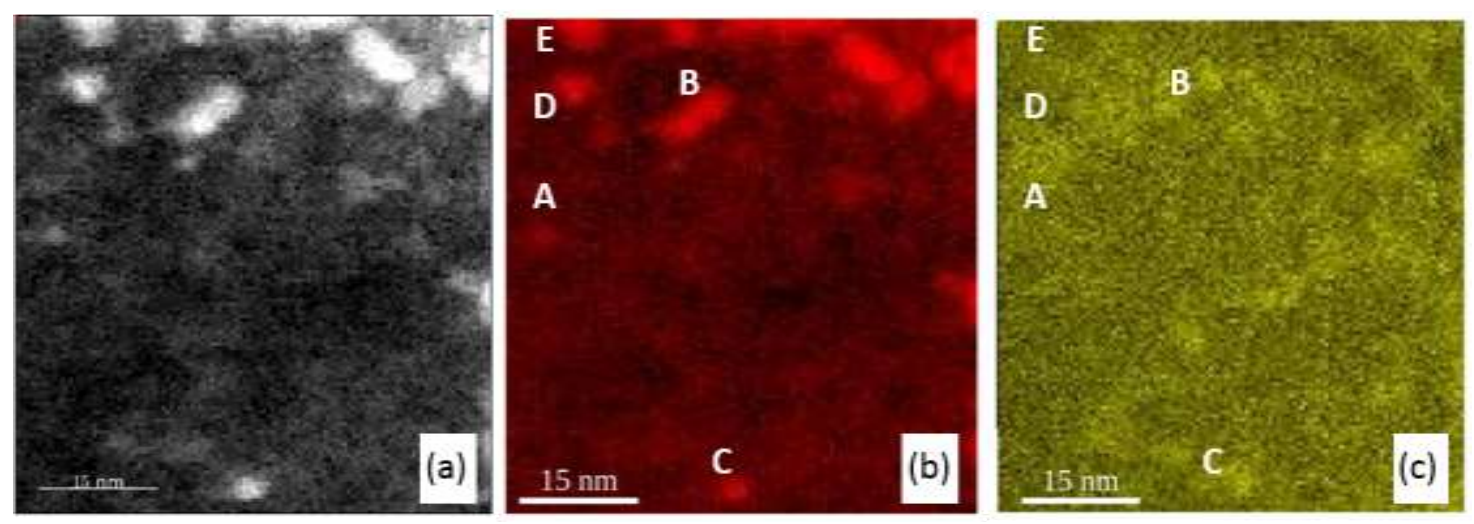

Figure S5. (a) High-angle annular dark field (HAADF) scanning TEM (STEM) image showing the area of the specimen which was used to acquire EELS spectrum-image from $\operatorname{In}(\mathrm{Zn}) \mathrm{P} / \mathrm{ZnS}$ core-shell NCs capped with ZnS shell using SILAR method. (b) In and (c) Zn STEM-EELS ele-

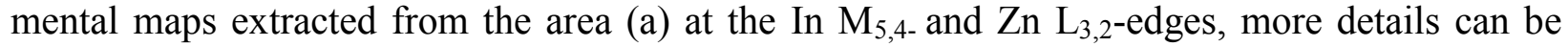
found in ref 3 and 4. Brighter areas in figure (a) indicate the positions of the NCs and they match well with the positions where In and $\mathrm{Zn}$ where found (seen as brighter areas in (b) and (c) respectively).

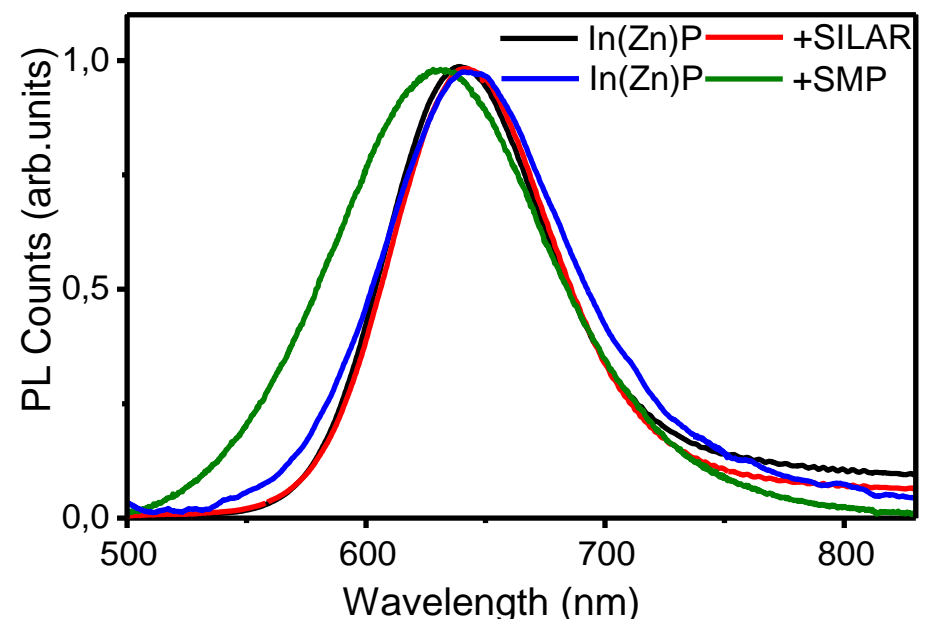

Figure S6. Comparison of PL from In(Zn)P core and core-shell NCs, for two different shell synthesis methods: SILAR and SMP. The cores were synthesized under the same conditions and they emit at the same wavelength. Both of them have a dominant band-to-band emission at 640 $\mathrm{nm}$ and both have a weak tail of counts from defect states extending to longer wavelengths. The PL spectrum of the cores later capped with the SMP method is slightly broader with a fwhm of $104 \mathrm{~nm}$ but has slightly smaller defect emission than the cores later used for capping with a shell using the SILAR method. The emission from the core-shell NCs capped with the SILAR method is centered at the same wavelength with a fwhm of $89 \mathrm{~nm}$ as PL of the cores. We mostly observed unchanged or slightly red shifted PL spectra after shell coating using the SILAR method. Note, that this means that any additional oxidation during the shell growth is insignificant and 
the size of the cores remains the same. The PL counts from the defects are reduced but still present even after the shell growth with the SILAR method. The PL of the core-shell NCs from the SMP method is blue-shifted compared with the core PL peak wavelength as expected from the NCs core size change. Considering also that the highest PLQY measured for NCs emitting at 630 $\mathrm{nm}$ with the SILAR grown shells was $30 \%$, while with the SMP grown shells $55 \%$, there is no evidence that this phosphate layer resulted in more effective $\mathrm{ZnS}$ shell growth or that it served as a good surface passivation. The phosphate layer might have however helped protecting the cores during their transfer from core synthesis to the shell reaction pot.

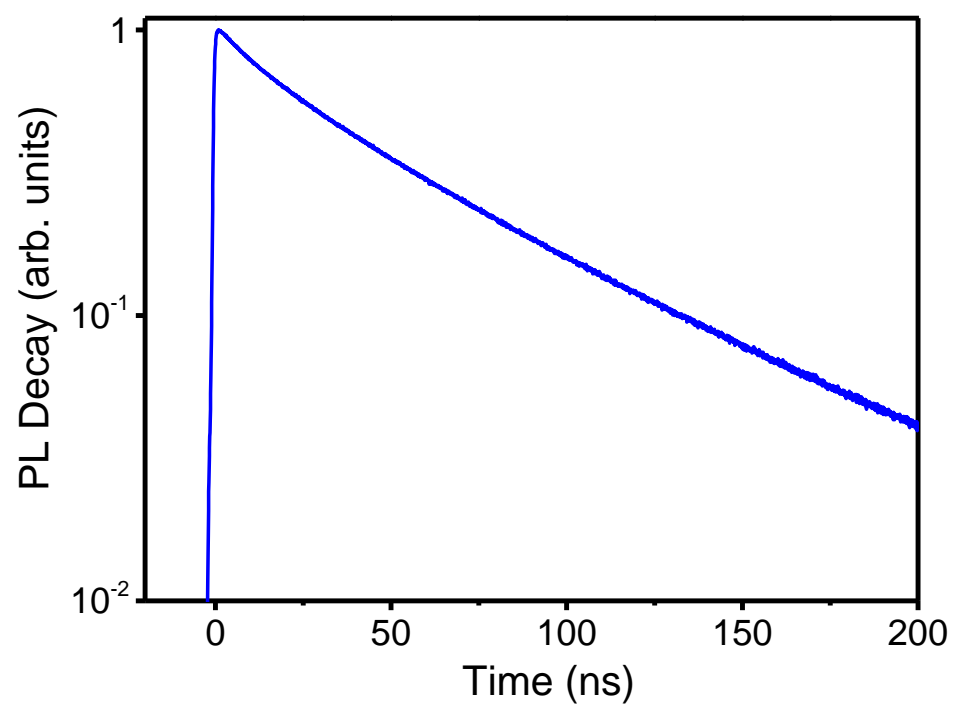

Figure S7. TRPL spectrum of In(Zn)P/ZnS NCs. The decay curve obtained by illuminating the NCs with a laser pulse of wavelength $405 \mathrm{~nm}$ at time zero. A very fast initial decay precedes almost single-exponential decay.

\section{Supporting Calculations and Table for the Shell Thickness}

1) Assuming that core/shell NCs is spherical and the diameter of core/shell NC (obtained from TEM image Figure $S 4 d$ ): $2 * R_{\text {core } / \text { shell }}=3.23 \pm 0.39 \mathrm{~nm}$.

2) Molar ratio of In to $\mathrm{Zn}$ in core/shell obtained from EELS is showed in Table S1. Same calculation is applied for other points for all below steps.

3) The density of InP is 4.81, the density of $\mathrm{ZnS}$ is 4.10 .

4) The molecular weight of $\mathrm{ZnS}: \mathrm{M} \cdot \mathrm{W} \cdot \mathrm{ZnS}=97.474 \mathrm{~g} / \mathrm{mol}$, the molecular weight of InP: $\mathrm{M} . \mathrm{W} \cdot \mathrm{InP}=145.792 \mathrm{~g} / \mathrm{mol}$,

Based on 2) and 3), the volume of $\mathrm{InP}$ at point $\mathrm{A}$ is 2.62 times that of $\mathrm{ZnS}$.

5) If the core radius in a core/shell $\mathrm{NC}$ is defined as $\mathrm{R}_{\text {core', }}$

Volume of InP core: $V_{\text {core' }}=4 / 3^{*} \pi^{*} \mathrm{R}_{\text {core' }}{ }^{3}$

Volume of $\mathrm{ZnS}$ shell: $\mathrm{V}_{\text {shell }}=4 / 3 * \pi *\left(\mathrm{R}_{\text {core/shell }}{ }^{3}-\mathrm{R}_{\text {core' }}{ }^{3}\right)$

Volume of InP/ZnS, $\mathrm{V}_{\text {core/shell }}=\mathrm{V}_{\text {core' }}+\mathrm{V}_{\text {shell }}$ 
6) Based on 4) and 5),

$\mathrm{R}_{\text {core' }}{ }^{3}=2.62 *\left(\mathrm{R}_{\text {core/shell }}{ }^{3}-\mathrm{R}_{\text {core' }}{ }^{3}\right)$,

Thus for point $A: R_{\text {core' }}=2.87 \mathrm{~nm}$,

7) The ZnS shell thickness in core/shell NC at point A: $d_{\text {shell }}=R_{\text {core/shell }}-R_{\text {core' }}=0.33 \mathrm{~nm}$.

8) The thickness of one monolayer $\mathrm{ZnS}$ is $0.31 \mathrm{~nm}{ }^{4,5}$

Thus, 1.06 monolayers worth of $\mathrm{ZnS}$ shell were coated on $\mathrm{In}(\mathrm{P}) \mathrm{Zn}$ core for point $\mathrm{A}$. The detailed calculation results for other points are summarized in Table S1.

Table S1. Relative atomic concentration of In and $\mathrm{Zn}$ and the shell thickness from five points (position A to E) in Figure S4b-c. Hartree-Slater cross-section from DM have been used for the calculation.

\begin{tabular}{|c|c|c|c|c|c|}
\hline & A & B & C & D & E \\
\hline In (at. \%) & 67.3 & 65.5 & 61.5 & 74.2 & 83.1 \\
\hline Zn (at. \%) & 32.7 & 34.5 & 38.5 & 25.8 & 16.9 \\
\hline In to Zn mol. ratio & 2.06 & 1.90 & 1.60 & 2.88 & 4.92 \\
\hline Core radius (nm) & 2.87 & 2.85 & 2.80 & 2.95 & 3.05 \\
\hline \begin{tabular}{c} 
Shell thickness (nm) \\
\hline $\begin{array}{c}\text { Number of monolay- } \\
\text { er(s) }\end{array}$
\end{tabular} & 0.33 & 0.35 & 0.40 & 0.25 & 0.15 \\
\hline
\end{tabular}




\section{References}

(1) Xi, L. F.; Cho, D.-Y.; Duchamp, M.; Boothroyd, C.; Lek, J. Y.; Besmehn, A.; Waser, R.; Lam, Y. M.; Kardynal, B. ACS Appl. Mater. 2014, 6, 18233-18242.

(2) Grabolle, M.; Spieles, M.; Lesnyak, V.; Gaponik, N.; Eychmüller, A.; Resch-Genger, U. Anal. Chem. 2009, 81, 6285-6294.

(3) Duchamp, M.; Xi, L.F.; Lam, Y. M.; Dunin-Borkowski, R. E.; Kardynal, B. Proceedings of the 18th International Microscopy Congress, Prague, Czech Republic, 7-12 September 2014, MS-1-O-2712 (2 pages).

(4) Duchamp, M.; Lachmann, M.; Boothroyd, C. B.; Kovàcs, A.; Haug, F.-J.; Ballif, C.; Dunin-Borkowski, R. E. Appl. Phys. Lett. 2013, 102, 133902.

(5) Virieux, H.; Le Troedec, M.; Cros-Cagneux, A.; Ojo, W-S.; Delpech, F.; Nayral, C.; Martinez, H., Chaudret, B. J. Am. Chem. Soc, 2012, 134, 19701-19708. 